OBETS. Revista de Ciencias Sociales

Vol. 16, no 2, 2021, pp. 435-448

ISSN: 1989-1385

https://doi.org/10.14198/OBETS2021.16.2.13

\title{
LA PRESENCIA DEL FACTOR INNOVADOR EN EL PRODUCTOR AGRARIO EN LA COMUNIDAD VALENCIANA. HERRAMIENTAS PARA FAVORECER LA INNOVACIÓN EN LA DIALÉCTICA RURAL-URBANA
}

\author{
THE PRESENCE OF THE INNOVATIVE FACTOR IN THE AGRICULTURAL PRODUCER IN THE \\ VALENCIAN COMMUNITY. TOOLS TO PROMOTE INNOVATION IN RURAL-URBAN DIALECTICS
}

\author{
Pau Pérez-Ledo \\ Universitat Politècnica de València, Spain \\ pabpele@esp.upv.es \\ https://orcid.org/0000-0001-6542-4179 \\ Mónica Segovia-Pérez \\ Universidad Rey Juan Carlos, Spain \\ monica.segovia@urjc.es \\ https://orcid.org/0000-0001-7346-2546
}

Cómo citar / Citation: Pérez-Ledo, P. y Segovia-Pérez, M. (2021) "La presencia del factor innovador en el productor agrario en la Comunidad Valenciana. Herramientas para favorecer la innovación en la dialéctica rural-urbana". OBETS. Revista de Ciencias Sociales, 16(2): 435-448. https://doi.org/10.14198/OBETS2021.16.2.13

\section{(C) 2021 Pau Pérez-Ledo, Mónica Segovia-Pérez}

Este es un artículo de acceso abierto distribuido bajo los términos de la licencia de uso y distribución Creative Commons Reconocimiento 4.0 Internacional (CC BY 4.0) https://creativecommons.org/licenses/by/4.0/deed.es

Recibido: 20/03/20. Aceptado: 18/01/21

\section{Resumen}

Este estudio sociológico se centra en la figura del productor agrario valenciano y su comportamiento frente a la innovación. Adoptar una actitud que favorezca la innovación depende de múltiples factores que influyen en la gestión de la empresa agraria. Este estudio utiliza los datos procedentes de una encuesta a productores agrarios valencianos que profundiza en los factores socioeconómicos que pueden decantar al productor agrario hacia una postura favorable a la innovación. Posteriormente, el estudio se centra en obtener un perfil tipo que represente al productor según sus características y las de su explotación, según su actitud innovadora y la orientación hacia factores influyentes, como son la orientación al mercado y la orientación al aprendizaje. Se observa una asociación positiva entre la actitud innovadora y el nivel de formación del agricultor y su proximidad a zonas urbanas, donde existe más acceso a conocimiento y servicios. El artículo concluye con la descripción de unos tipos ideales weberianos sobre agricultores basándose en su orientación hacia la innovación.

\begin{abstract}
This sociological study focuses on rural innovation. Farmers are the main actors of innovation in the agricultural sector. This study focuses on the socio-economic profile of the Valencian farmer and his/her attitude towards innovation. Adopting a position inclined to innovate depends on multiple factors that influence bussiness management. This paper uses data from a farmers' survey carried out in the Valencia region, that considers the socioeconomic factors that make farmers take a position favorable to innovation. Later, the study focuses on a typical profile of innovative producers, according to their characteristics, taking into account market orientation, learning orientation and innovative attitude. The results show a positive contribution of level of education and location in urban areas (access to knowledge and services) to the innovative attitude.
\end{abstract}

Keywords: Innovation, agriculture, education, rural sociology.

Palabras clave: Innovación, agricultura, educación, sociología rural. 


\section{Extended abstract}

This sociological study focuses on rural innovation. Farmers are the main actors of innovation in the agricultural sector. This study focuses on the socio-economic profile of the Valencian farmer and his/her attitude towards innovation. Adopting a position inclined to innovate depends on multiple factors that influence business management. This paper uses data from a farmers' survey carried out in the Valencia region, that considers the socioeconomic factors that make farmers take a position favorable to innovation. Later, the study focuses on a typical profile of innovative producers, according to their characteristics, taking into account market orientation, learning orientation and innovative attitude. The results show a positive contribution of level of education and location in urban areas (access to knowledge and services) to the innovative attitude.

The study of innovation is not new in the analysis of the social sciences applied to agriculture and the rural world. However, few studies integrate the analysis of the behavior of the main subject of the innovation: the farmer. And this is precisely one of the main contributions of this research. On the other hand, small companies appear underrepresented in statistics or official studies and there are not enough previous studies on their circumstances and the impact that this has on the invention. Likewise, the study of the case of the Valencian agricultural producer and his behavior in the face of innovation can shed light on many questions of innovative behavior that can be transferred to other contexts. Likewise, the analysis is framed in a time point close to the end of the last existing agricultural census, which makes it possible to clarify the present attitudes of the farmers of the Valencian Community. The different statistical analyzes used will lead us to know the key variables that should guide public action to favor innovation in this area, improving the capacity of public policies in the field of agricultural innovation, helping to ease the tension between rural and rural areas. the urban.

In general terms, this research has provided knowledge with a new point of reference to advance in the study of innovation in the Valencian agricultural sector.

The results of the research show the socioeconomic characteristics of the producer and his exploitation that most favor innovative behavior, understood as the combination of three components: market orientation, learning orientation and innovative attitude. In our survey there is a high presence of farmers with an innovative attitude, which we do not consider necessarily represents the Valencian farmer, but does denote what characteristics the most innovative farmers have.

It is interesting to note that the innovative attitude is closely associated with the level of training: the higher the education, the better the predisposition the producer shows to adopt an innovative attitude. Therefore, the results emphasize the need for professional business managers, which in rural areas today is a handicap. These are aspects that educational and rural development policies should take into account.

All these factors indicate the attempt by the sector to alleviate one of the structural problems of the sector such as part-time agriculture. Throughout the decades, the drift of agriculture towards this productive model allowed maintaining the smallholder structure and the producer adopting a different position with respect to exploitation, assuming other occupations as his main activity. Smallholders are also an aspect that is associated with partial agriculture, and with them the deprofessionalization of the sector. Assuming the leasing regime for the farm may be a reflection of how it is trying to reach a size necessary to take advantage of economies of scale, which obviously requires a professional producer entirely dedicated to the activity. Part-time agriculture also resulted in the rise of organizations whose objective was to provide services as a result of the deprofessionalization of the sector, since the producer saw it possible to carry out agricultural activity combining it with activity in other sectors. On this aspect, belonging to an organization is an aspect that, in our research, favors innovation, especially in the case of cooperatives or SATs. In this way, they are aligned with the 2020 European Strategy with the establishment of interorganizational links. Thus, we can verify that the Valencian organizational structure was born and flourished with one objective, and today it is an extremely useful factor to maintain Valencian agriculture in the modern economy. However, we must not forget that partial agriculture worked at the time, giving way to problems like this, which are a consequence of the structural deficiencies of the sector, but at present we record the need to professionalize Valencian agriculture.

The study shows that the agricultural producers most involved in the activity are those who really adopt a fruitful attitude to ensure that the sector is fully integrated into the current economic dynamics. Thus, the innovative producer is one who shows involvement in his activity and demonstrates it by dedicating more than half of his time to exploitation. Innovative behavior is also favored by the fact that all or part of the farm is under lease. In the same way, innovation is favored by having salaried labor.

On the other hand, depending on the production orientation, we see that the farms that are dedicated to citrus or horticultural crops tend to be in the innovative group. This impression can be reinforced by the fact that they are highly market-oriented sectors, with a notable export side. But, on the other hand, farms that have an irrigation infrastructure are also farms with an innovative predisposition. This fact may highlight that coastal agriculture differs from inland agriculture, with the coastal area appearing to be more integrated in the current economic dynamics. This suggests the need to adopt rural development policies that revitalize economic diversification in inland areas, also taking into account the challenge of sustainability.

Finally, in the innovative group there are very present farms that belong to obtain an annual gross margin of more than $€ 50,000$ per year. This trend shows the financial insecurity suffered by the sector and illustrates the fact that adopting innovations is associated with the investment of money. We do not mean by this that being small condemns the farm to be weakly innovative, since it is a set of characteristics that end up influencing innovative behavior.

In summary, it has been observed that the structural deficiencies that were the object of study at the end of 
the 20th century are still present in Valencian agriculture, although it is true that a timid evolution can be observed in order to alleviate them. With all this, the content of this work shows the effort by the innovative Valencian producer to face the traditional problems that afflict the sector. Education appears as one of the key factors on which we must continue to influence to make the sector viable. This work can shed light when it comes to carrying out active policies to promote agricultural innovation since it clearly indicates the variables that can be influenced to improve the effectiveness of public efforts in the sector.

\section{INTRODUCCIÓN}

La innovación se ha convertido en una pieza clave para generar valor de una manera más eficaz y eficiente a la hora de satisfacer las nuevas exigencias sociales y medioambientales en un mundo en constante transformación (Yagüe-Perales, R. M., Perez-Ledo, P., \& March-Chorda, I., 2020; De la Fuente Cabrero, C., Segovia-Pérez, M., \& Figueroa-Domecq, C., 2014). Como el resto de los sectores, el sector agroalimentario, inmerso en un escenario que cambia con rapidez, ha tenido que enfrentarse a sus propios retos. Estructuralmente, se considera un sector con influencia en el desarrollo del territorio, por sus funciones de suministrador de alimentos y su alta ocupación de éste (López-García et al, 2012). No obstante, y pese a las grandes transformaciones que ha asumido a lo largo de los tiempos, tradicionalmente la literatura identifica al sector agrario como un segmento con baja intensidad innovadora (Connor y Chiek, 1997). No obstante, estudios recientes refutarían los resultados anteriores, mostrando que, en el caso específico del sector agroalimentario valenciano, éste posee una intensidad innovadora mayor que otros sectores del mismo territorio (Alba, 2012), aunque en conjunto, la innovación en esta zona sea menor comparado con el conjunto nacional. Sin embargo, un análisis más pormenorizado del entorno de la Comunidad Valenciana diferenciando entre el sector primario o la industria agroalimentaria, pone de relieve que el primero (sector primario) acaba resultando de baja intensidad innovadora en comparación con la industria agroalimentaria en dicha comunidad. Pese a estos resultados, las investigaciones también han puesto de relieve que los vínculos intersectoriales provocan una innovación indirecta -o incorporada en los productos/ servicios que el sector adquiere-, proveniente de otros sectores económicos. Estudios recientes (Alba et al, 2010) indican que la innovación indirecta es importante en el sector primario, mucho más que en la industria agroalimentaria, y que, además, esta importación de conocimiento se realiza predominantemente desde otros territorios del país.

No obstante, cabe preguntarse el motivo de esta baja intensidad innovadora en el sector agrario, ya que no deja de ser curiosa la diferencia respecto a la industria alimentaria (ambos ubicados en la Comunidad Valenciana). ¿Se debe a factores internos, derivados de la propia estructura del sector, que rigidiza o dificulta la incorporación de innovaciones? ¿Es debido a una percepción derrotista por parte del productor agrario, responsable de la iniciativa innovadora en su explotación? Si esto fuera cierto, ¿qué influye en la percepción del productor? ¿Factores sociológicos, individuales...? ¿O es quizá la compleja estructura del mercado la que limita el margen de maniobra de un productor que realmente está motivado? La presente investigación atiende a los factores que la literatura identifica como claves en el proceso de toma de decisiones relativas a innovación con el fin de elaborar el perfil "tipo" del productor valenciano innovador. El objetivo principal de la investigación es identificar las características que definirían el perfil del productor agrario valenciano innovador, analizando información obtenida de primera mano mediante una encuesta a los productores.

El estudio de la innovación no es nuevo en el análisis de las ciencias sociales aplicadas a la agricultura y el mundo rural. Sin embargo, pocos estudios integran el análisis del comportamiento del sujeto principal de la innovación: el agricultor. Y precisamente es ésta una de las principales aportaciones de esta investigación. Por otro lado, las empresas de pequeñas dimensiones aparecen infra-representadas en las estadísticas o estudios oficiales y no existen suficientes estudios previos sobre sus circunstancias y el impacto que ello tiene en la invención. Asimismo, el estudio del caso del productor agrario valenciano y su comportamiento frente a la innovación puede arrojar luz sobre muchos 
interrogantes del comportamiento innovador que pueden ser trasladables a otros contextos. Asimismo, el análisis se encuadra en un momento temporal cercano al final del último censo agrario existente, lo que permite esclarecer las actitudes presentes de los agricultores de la Comunidad Valenciana. Los diferentes análisis estadísticos utilizados nos conducirán a conocer las variables clave que deben guiar la acción pública para favorecer la innovación en este ámbito, mejorando la capacidad de las políticas públicas en el ámbito de la innovación agraria ayudando a flexibilizar la tensión entre el ámbito rural y el urbano.

\section{MARCO TEÓRICO}

La innovación es definida como un proceso mediante el cual, a partir del reconocimiento de la demanda, que puede venir de una organización y/o del mercado, se genera una serie de invenciones, cristalizadas o bien en los productos, la comercialización o los procesos o métodos organizativos, que, posteriormente, serán adoptados y generalizados como innovaciones (de Cádiz, D. C. T. G., Montejo, M. Z., \& Ramiro, C. J. M., 2020). En el caso específico de la innovación agraria ésta posee una serie de características distintivas con respecto a otros sectores que condicionan la misma y que se resumen en las siguientes: el medio principal e insustituible de producción es la tierra; el medio de producción son organismos vivos, sujetos a cambios biológicos y naturales; la producción tiene un carácter cíclico y estacional; la producción presenta varias formas de propiedad y; posee una fuerza de trabajo con características muy específicas (de Cádiz, D. C. T. G., Montejo, M. Z., \& Ramiro, C. J. M., 2020; Cordovés Torres Gómez de Cádiz, D., Franch León, K., Zenea Montejo, M., Díaz Fernández, I., \& Toscano Ruiz, D. F., 2017). Asimismo, en el sector agrario, la innovación puede ocurrir como consecuencia de los vínculos intersectoriales, interorganizacionales (mediante cooperativas) o interpersonales produciendo una innovación indirecta. En este caso, es importante el concepto de "Sistema de Conocimiento e Innovación Agrícola" (AKIS- "Agricultural Knowledge and Innovation System") que se utiliza para describir cómo las personas y las organizaciones se unen para generar, compartir y utilizar el conocimiento y la innovación para la agricultura, promoviendo el aprendizaje mutuo (European Union, 2019). No hay que olvidar que la base del desarrollo territorial/rural ha sido la interacción social y la creación de redes de personas y grupos dispuestos a colaborar y comprometerse con el colectivo (Savall, N. V., Pizarro, J. E., \& López, D. E. V.,2020).

Por otro lado, la innovación ha permitido la transición desde territorios con una supremacía agraria hacia la diversificación productiva (Aguilar Criado, E., Amaya Corchuelo, S., \& López Moreno, I., 2016, Segovia-Pérez, M., Santero Sánchez, R., Domecq, C. F., \& Núñez, R. B. C., 2019). Por ello, la innovación ha sido un elemento crucial para el desarrollo sostenible del sector agroalimentario a lo largo del tiempo como estrategia para dar respuesta a las nuevas exigencias de la globalización, la propia sociedad y los nuevos tipos de consumidores (green consumers) (Albisu Aguado, L. M., 2019, Pomering, A.,2017). De hecho, desde la Unión Europea, en su Estrategia Europea 2020 para un crecimiento integrador, considera que la "innovación en las zonas rurales puede desempeñar un papel importante en la estimulación del crecimiento económico ecológico y socialmente inclusivo, mitigando el aislamiento geográfico y evitando la marginación económica y social" (European Union, 2019). En este contexto, una de las tácticas del Programa de Desarrollo Rural en Europa a lo largo del tiempo ha sido la de generar estrategias para crear valor a partir de la especialización, la búsqueda de certificaciones de calidad y, por supuesto, la innovación (Fernández Zarza, M., Amaya Corchuelo, S. y Aguilar Criado, E., 2019, Amaya-Corchuelo, S., Fernández Zarza, M., \& Aguilar Criado, E., 2018). Esta constante transformación se ha conocido como la nueva ruralidad (Aguilar, E., AmayaCorchuelo, S., y López-Moreno, I. 2016).

Junto a las cuestiones expuestas, uno de los aspectos fundamentales en los que se incide a la hora de determinar la innovación es la importancia de la persona que dirige la propiedad (Salas Arbeláez, L., García Solarte, M., \& Azuero Rodríguez, A. R., 2018) siendo el actor social que realmente la promueve y estimula entre sus equipos. Así, la Unión Europea considera que el capital humano y social son esenciales para conseguir una innovación rural exitosa, dado que son los actores mediante sus habilidades y actitudes innovadoras los que pueden iniciar actividades, generar vínculos, incentivos, valores compartidos y normas que sustenten el proceso innovador (European Union, 2019). Por tanto, es crucial el desarrollo de 
investigaciones centradas en las actitudes, aptitudes y comportamientos innovadores de los propios agricultores. No obstante, la adopción de una actitud que favorezca la innovación depende de múltiples factores que influyen en la gestión de la empresa agraria (Pérez Ledo, P., 2018). Según de Cádiz, D. C. T. G., Montejo, M. Z., \& Ramiro, C. J. M. (2020) las características cualitativas de los actores sociales que protagonizan la realización de la innovación agraria influyen directamente en el resultado de dicho proceso. Estas características son su cualificación y experiencia en la actividad, así como el conocimiento de la tecnología y su resultado productivo.

Asimismo, según Yagüe-Perales, R. M., et al. (2020) las innovaciones son el vehículo mediante el cual la ciencia se transforma para incorporarse a las empresas y crear valor. Por ello, el empresario tiene que mostrar una orientación favorable al aprendizaje para modificar su actividad y acometer innovaciones de todo tipo (producto, proceso, organización, función, etc).
Adicionalmente, los estudios resaltan la importancia de adoptar una estrategia empresarial basada en la orientación al cliente que, combinándola con una actitud innovadora, mejora la competitividad de las empresas y su integración en el entorno económico. Se puede aceptar el hecho de que existe una retroalimentación entre actitud innovadora y orientación al mercado, de manera que la comunicación empresa-cliente fomenta la puesta en marcha por parte de la empresa de los productos/servicios que el cliente demanda. Dicho de otra manera, incorporar en la planificación empresarial el conocimiento de las necesidades de los clientes, incide en innovaciones más acertadas, con mayor probabilidad de éxito en el mercado, y por tanto menor riesgo para el emprendedor que se encarga de introducirlas. De inmediato toman protagonismo factores como el conocimiento o el mercado. El eslabón que une ambos es el innovador o emprendedor que ha de tener sendos vínculos con el conocimiento y con su cliente, según el esquema de la Figura 1.

Figura 1. Aplicación de innovaciones por parte del responsable.

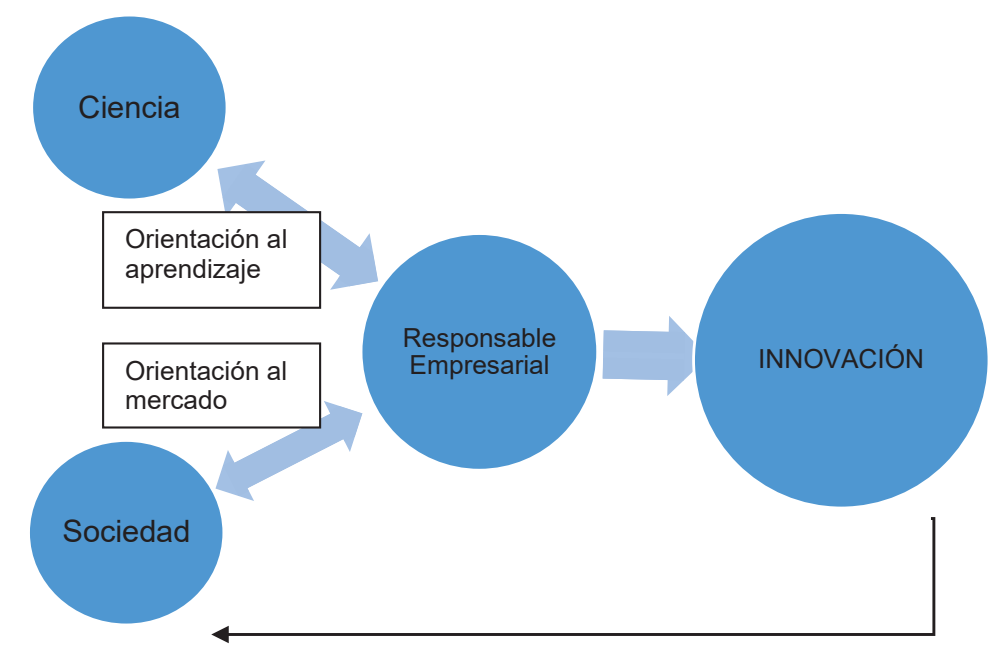

Fuente: Elaboración propia.

Teniendo en cuenta la relevancia histórica, cultural y económica del sector agrario en la Comunidad Valenciana, el estudio del caso del productor agrario valenciano y su comportamiento frente a la innovación puede arrojar luz sobre todas estas cuestiones en un contexto similar a otros entornos y desde un punto de vista empírico. De hecho, muchos de los componentes globales que favorecen o entorpecen la innovación agraria aparecen en esta comunidad. De entre todos los rasgos que diferencian la agricultura valenciana de sus homónimas, el minifundismo ha sido uno de los temas de debate más presentes en la literatura. Es tan inherente al campo valenciano, que un $40 \%$ de las explotaciones poseen menos de una hectárea. Bajo estas circunstancias la tierra se ha convertido en un factor de producción escaso en los planteamientos 
empresariales. Las explotaciones no han podido evolucionar hacia un tamaño mínimo que permita aprovechar la economía de escala. En el año 2009 cerca del 54\% de las explotaciones poseían menos de dos hectáreas. La evolución entre los Censos de 1999 y 2009 -último censo existente- muestra un descenso en el número de explotaciones con menos de una hectárea y un aumento en el número de explotaciones de mayor tamaño. La explicación puede estar en el descenso del número total de explotaciones, lo que indicaría un abandono de la agricultura por parte de algunos agricultores; de esta forma, estas tierras, que se quedan sin explotar, son adquiridas por el resto de las explotaciones, que aumentan su tamaño.

La literatura coincide en enmarcar otra particularidad del sector valenciano, que es causa y consecuencia de la agricultura a tiempo parcial. Arnalte (1989) denominaba a este proceso la "desactivación de explotaciones", refiriéndose al cambio paulatino hacia la externalización de gran parte de las tareas agrícolas adentrándose en un modelo de organización productiva, típica de regadío, que permite el manejo de una mayor superficie por cada persona ocupada. Este modelo va aparejado con la agricultura a tiempo parcial y permite mantener la estructura minifundista de las explotaciones, en lugar de promover la concentración para alcanzar mayores tamaños.

En la actualidad vemos que los problemas que describieron los economistas y geógrafos valencianos para la agricultura valenciana en los años ochenta y noventa del siglo XX persisten. Sin embargo, según pasan los años se puede apreciar una evolución tímida en los indicadores que muestran el esfuerzo del sector en pro de alcanzar un equilibrio. Por ejemplo, la estructura minifundista se ha visto modificada por la dinámica del sector, que ha permitido que las explotaciones del interior tiendan a reestructurarse. La pérdida de población ocupada que se ha experimentado con los años ha permitido cierta concentración de las explotaciones, por lo que se puede observar un aumento del tamaño medio. En términos generales, la Comunidad Valenciana ha perdido en 10 años casi el 47\% de explotaciones, según el Censo Agrario (1999-
2009, INE). No obstante, la agricultura litoral ha mantenido su alto grado de minifundismo.

A partir de este contexto, existe una carencia de estudios preliminares sobre la comunidad valenciana que deja múltiples interrogantes a nivel de productor; ¿qué percepción tiene de la innovación? ¿cómo influyen los factores como la orientación al mercado en la actitud innovadora?; o por el contrario ¿influye la orientación al mercado en la actitud innovadora? En definitiva, ¿qué determina el comportamiento innovador en el productor agrario valenciano?. Y respecto a los factores más relacionados con las características del individuo y con las de su explotación, ¿es de suponer que también ejercen influencia sobre la actitud innovadora? Hay ciertos factores que favorecen una actitud innovadora sea cual sea el sector al que se aplique, ipodrían extrapolarse al sector agrario valenciano? ¿cómo influiría, por ejemplo, la edad o el sexo en la actitud innovadora?

\section{METODOLOGÍA}

La metodología empleada en el presente trabajo se basa en los datos obtenidos mediante una encuesta que fue realizada durante el segundo semestre del año 2011, basada en el último censo agrario publicado en el año 2009 por el INE. Tras unos meses de recogida de información se creó una base de datos codificada, con 253 encuestas válidas.

La encuesta tenía como objetivo recopilar información acerca de factores que pueden influir en el comportamiento innovador como: las características sociodemográficas del productor (factoresindividuales), de su explotación (factores organizativos) y de todos los elementos que puedan constituir una definición del comportamiento innovador o influir en la toma de decisiones relativas a la innovación.

Por lo que se refiere a las variables socioeconómicas para segmentar a la población en la Tabla 1 se pueden ver el conjunto de factores individuales y organizativos que pueden influir en el comportamiento innovador del agricultor. 
Tabla 1. Listado de características individuales y organizativas.

\begin{tabular}{|c|c|}
\hline Individuales & Organizativos \\
\hline $\begin{array}{l}\text { - Edad } \\
\text { - Sexo } \\
\text { - Porcentaje tiempo dedica a la } \\
\text { explotación } \\
\text { - Formación } \\
\text { - Años de trayectoria profesional } \\
\text { - Año desde el que es titular de } \\
\text { la explotación } \\
\text { - Titularidad compartida con el } \\
\text { cónyuge } \\
\text { - Pertenencia a organizaciones }\end{array}$ & $\begin{array}{l}\text { - Tamaño de la explotación } \\
\text { (Hectáreas cultivo/cabezas) } \\
\text { - Año desde el que existe la } \\
\text { explotación } \\
\text { - Ayuda familiar } \\
\text { - Contrata asalariados } \\
\text { - Régimen de tenencia } \\
\text { - Sistema de riego } \\
\text { - Agricultura ecológica } \\
\text { - Destino de la producción } \\
\text { - Cliente preferente y acuerdos } \\
\text { - contractuales } \\
\text { - Aargen Bruto de la explotación } \\
\text { Actividades complementarias }\end{array}$ \\
\hline
\end{tabular}

En cuanto a los factores que resumen el comportamiento innovador del productor agrario valenciano y basado en Pérez Ledo, P. (2018), se definieron tres grandes grupos, que son:

- la orientación al mercado (OM)

- la orientación al aprendizaje (OA)

- la actitud innovadora (AI)

Estos tres factores fueron construidos como una representación y/o conceptualización abstracta del comportamiento innovador. Para su medición se siguieron tres pasos que se explican a continuación. El primer paso fue asignar a cada factor (constructo ${ }^{1}$ ) una serie de afirmaciones o ítems para que el encuestado manifestará su grado de acuerdo o desacuerdo. Para ello, se utilizó la escala Likert, que es la técnica más utilizada para medir aspectos de la conducta, como puede ser la satisfacción de un cliente, ya que el procedimiento de Likert es muy intuitivo. Así pues, nuestra encuesta a agricultores valencianos diseñó una serie de afirmaciones sobre cada uno de los tres factores (constructos) -su orientación al mercado, su orientación hacia el aprendizaje y su actitud innovadora-. El encuestado reflejó su grado de acuerdo puntuando cada una de esas afirmaciones en una escala de 1 a 7 , siendo 1 "completamente en desacuerdo" y 7 "completamente de acuerdo". En cada una de las afirmaciones se procuró reflejar un aspecto o dimensión

1 El constructo representa un concepto abstracto cuya definición es difícil de establecer y que precisa de medidas indirectas para su estudio (Pérez-Gil et al. 2000). La técnica estadística para la construcción de estos constructos se explica en este apartado. del factor (constructo) que estábamos estudiando. Por ejemplo, el factor (constructo) "Actitud Innovadora" puede medirse según el nivel de acuerdo que refleje el encuestado respecto a esta afirmación:

\section{"Adoptar innovaciones es una decisión útil"}

Afirmación que puede traducirse, con bastante seguridad, como el grado de asociación que establece el encuestado entre innovar y utilidad. Así pues, estamos midiendo la actitud innovadora mediante una de sus facetas: la utilidad. Pero previsiblemente la actitud innovadora no posee exclusivamente ese significado así que, en base al razonamiento teórico, decidimos incluir otras afirmaciones para reforzar la medición del constructo. Así, por ejemplo, en la Tabla 2. mostramos una serie de afirmaciones a título de ejemplo que vendrían a reflejar el constructo "orientación al mercado".

Tabla 2. Ejemplo de variables Likert para medir la "Orientación al mercado".

\begin{tabular}{|c|c|c|c|c|c|c|c|c|}
\hline & & & & & & & & \\
\hline $\begin{array}{l}\text { Sigo las orientaciones } \\
\text { de calidad que } \\
\text { transmite el cliente. }\end{array}$ & 1 & 2 & 3 & 4 & 5 & 6 & 7 & NS \\
\hline $\begin{array}{l}\text { Cada año busco } \\
\text { nuevos clientes }\end{array}$ & 1 & 2 & 3 & 4 & 5 & 6 & 7 & NS \\
\hline $\begin{array}{l}\text { Los clientes me orientan sobre } \\
\text { las variedades a plantar }\end{array}$ & 1 & 2 & 3 & 4 & 5 & 6 & 7 & NS \\
\hline $\begin{array}{l}\text { Mi preocupación por la } \\
\text { calidad me da una ventaja } \\
\text { sobre otras explotaciones. }\end{array}$ & 1 & 2 & 3 & 4 & 5 & 6 & 7 & NS \\
\hline $\begin{array}{l}\text { Mi preocupación por ofrecer } \\
\text { productos más baratos } \\
\text { me da una ventaja sobre } \\
\text { otras explotaciones }\end{array}$ & 1 & 2 & 3 & 4 & 5 & 6 & 7 & NS \\
\hline
\end{tabular}

Fuente: Elaboración propia.

El siguiente paso en la medición de los factores que influyen en el compartiendo innovador a partir de los constructos (factores), consistió en simplificar la información, perdiendo la menor cantidad de información posible, con el objetivo de medir los factores (constructos). La técnica seleccionada para este propósito fue el análisis de componentes principales (ACP). La técnica estudia la estructura de las interrelaciones dadas entre un gran número de variables, con el fin de hallar dimensiones subyacentes, conocidas como componentes (en realidad, los indicadores sintéticos de los tres constructos definidos por una serie de preguntas de la encuesta). La técnica 
da como resultado una serie de nuevas variables, que serán la representación del componente hallado en la estructura de las variables, es decir, los constructos (o factores). El ACP es un procedimiento estadístico que tiene como objetivo el análisis de la variación total que una variable comparte con otras variables, para poder resumir la información contenida en el conjunto total de variables interrelacionadas. La ventaja que otorga el $\mathrm{ACP}$ es que reduce la información disponible creando nuevas variables que son combinaciones lineales del conjunto inicial perdiendo la menor cantidad posible de la variabilidad inicial. La técnica permitió definir en forma de componentes o variables sintéticas, los tres constructos: Orientación al Mercado, Orientación al Aprendizaje, y Actitud Innovadora.

En nuestro caso, diez variables Likert de la encuesta fueron sintetizadas en dos componentes correspondientes a los constructos Orientación al Mercado y Orientación al Aprendizaje, que en conjunto explicaban un $57 \%$ de la varianza original de las variables originales. Se definió el componente correspondiente al constructo Actitud Innovadora a partir de seis variables Likert de modo que dicho componente principal explicaba el $60 \%$ de la varianza inicial de las variables iniciales.

Figura 2. Obtención de variables representativas de los constructos.

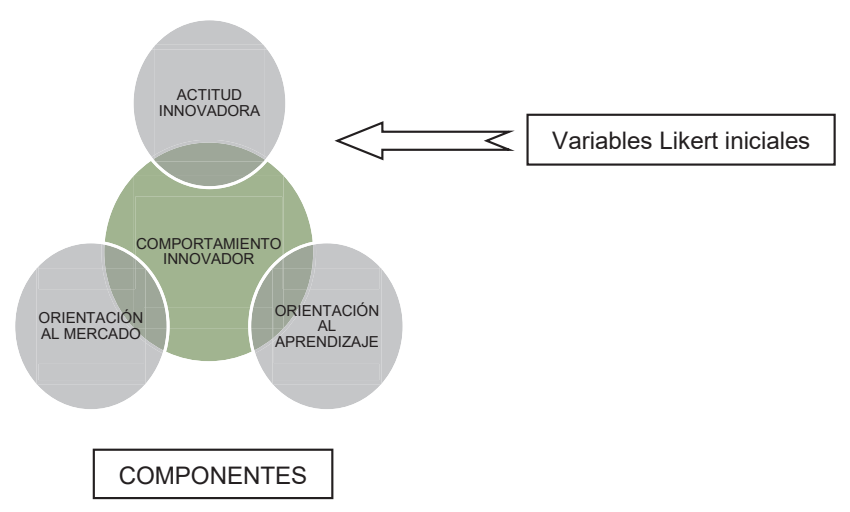

Fuente: Elaboración propia.

Una vez definidos los factores, reflejados en la Figura 2, el tercer y último paso fue el establecimiento de la relación entre ellos, con el fin de confirmar las dimensiones que componen la conducta innovadora.

Una vez obtenidas las variables representativas de los factores (constructos) de estudio se aplicaron procedimientos estadísticos destinados a conocer si existía diferencia entre las puntuaciones medias de diferentes grupos respecto a los mismos. El uso de las técnicas multivariantes permitió realizar un análisis conjunto de varias variables con el fin de encontrar un modelo que explique el comportamiento innovador, por lo que se aplicaron modelos de regresiones múltiples incorporando en cada uno de ellos, como variables exógenas, las características individuales y organizativas que figuran en la Tabla 1.

\section{RESULTADOS}

\subsection{Características individuales y organizativas de los agricultores valencianos}

Los datos obtenidos mediante la encuesta constatan que la muestra en general es un poco más joven de lo que plasman las estadísticas agrarias oficiales; la media de edad se sitúa en torno a los 50 años, lo que sigue reflejando una población relativamente envejecida. La masculinización del mundo rural se deja sentir en los resultados, que son algo más extremos de lo que cabía esperar. Ciertamente el número de mujeres encuestadas es bajo, un $9 \%$ del total, lo cual se debe posiblemente al haber tomado como base una muestra de afiliados a organizaciones agrarias y cooperativas.

En lo que respecta al perfil del encuestado, se trata de un productor en general implicado en su explotación, que dedica más del $50 \%$ de su tiempo a la explotación (un $74 \%$ de ellos lo hace). La formación con la que cuenta oscila entre el graduado escolar y formación a nivel de bachillerato o FP, aunque también hay un $13 \%$ de titulados universitarios. En cuanto a su experiencia como titular de explotaciones agrarias, ésta es amplia, de media 22 años, con la particularidad de que también son muchos los años que lleva gestionando la explotación. Eso sí, compaginándolo a lo largo de su vida con el trabajo en otros sectores distintos al agrario.

Las cooperativas o SATs aglutinan a la mayoría de encuestados que declararon pertenecer a alguna asociación agraria, un $67 \%$, así como las comunidades de regantes, un $45 \%$, y las organizaciones profesionales agrarias, un $29 \%$.

En la Comunidad Valenciana la producción agrícola vegetal supone la mayor parte de la producción agraria final. La muestra sigue un patrón similar presentando un $80 \%$ de explotaciones que son agrícolas, un $15 \%$ 
que también tienen ganadería y finalmente un 3'5\% de explotaciones mayoritariamente ganaderas. También hay representación próxima a la realidad agraria en los cultivos, con los cítricos en el primer puesto por superficie, horticultura, viñedo, olivar y almendro entre otros caracterizando la diversidad agraria de la Comunidad. Por su parte el ganado porcino es la cabaña ganadera más presente en la muestra, seguida del sector avícola.

En cuanto a la mano de obra que trabaja en la explotación aproximadamente la mitad de los encuestados declara contratar asalariados. Otra mitad, aproximadamente, también declara hacer uso de familiares para las tareas agrarias.

El riego por goteo está muy extendido entre la muestra ya que el $60 \%$ lo tiene implantado en su terreno. Otro factor que define a la explotación es el destino de la producción; aquí la cooperativa vuelve a destacar, obviamente, pero también se constata el destino a comercios mayoristas. Esta no es una característica excluyente y los encuestados hacen uso de varias vías simultáneas para dar salida a sus productos. El margen bruto que engloba a casi el $60 \%$ de las explotaciones se encuentra en el rango comprendido entre 20.000 y $50.000 € /$ año.

\subsection{La opinión de los agricultores valencianos sobre los factores innovadores}

A la hora de analizar los factores innovadores de los agricultores valencianos se separaron aquellas cuestiones que tienen que ver con la orientación al mercado y al aprendizaje de los agricultores, por un lado, y las que reflejan una actitud positiva hacia el hecho de innovar, por el otro. En cierta medida, siguiendo el esquema de la Figura 1, la primera categoría representa la propensión del agricultor para captar información del exterior y ser capaz de asimilarla, mientras que la segunda ya denota el carácter más o menos innovador del agricultor. La siguiente tabla muestra los estadísticos descriptivos de cada una de las afirmaciones en escala de Likert que componen cada uno de estos factores (orientación al mercado y al aprendizaje; actitud innovadora).

Tabla 3. Estadísticos descriptivos de las variables Likert.

\begin{tabular}{|c|c|c|c|c|}
\hline & VARIABLE & $\mathrm{N}$ & Media & $\begin{array}{l}\text { Desv. } \\
\text { típ. }\end{array}$ \\
\hline \multirow{11}{*}{$\begin{array}{c}\text { ORIENTACIÓN } \\
\text { AL MERCADO } \\
\text { Y AL APRENDIZAJE }\end{array}$} & Sigo las orientaciones de calidad que transmite el cliente & 214 & 5,77 & 1,50 \\
\hline & Cada año busco nuevos clientes & 191 & 3,42 & 2,26 \\
\hline & Los clientes me orientan sobre las variedades a plantar & 197 & 3,43 & 2,23 \\
\hline & Mi preocupación por la calidad me da una ventaja sobre otras explotaciones & 221 & 5,22 & 1,87 \\
\hline & $\begin{array}{l}\text { Mi preocupación por ofrecer productos más baratos me } \\
\text { da una ventaja sobre otras explotaciones }\end{array}$ & 199 & 3,03 & 1,97 \\
\hline & La satisfacción del cliente es el principal objetivo de mi empresa & 218 & 5,72 & 1,68 \\
\hline & Me gusta leer revistas sobre nuevos cultivos o métodos que podría introducir & 235 & 5,56 & 1,64 \\
\hline & Me gusta asistir a ferias, cursos o jornadas para conocer nuevas ideas & 238 & 5,65 & 1,56 \\
\hline & Intercambio experiencias con otros agricultores & 239 & 5,95 & 1,23 \\
\hline & Cuando una nueva técnica/producto no da resultados analizo las causas del fallo & 233 & 5,67 & 1,49 \\
\hline & Me siento corresponsable de los fallos en mi explotación & 241 & 6,05 & 1,25 \\
\hline \multirow{6}{*}{$\begin{array}{l}\text { ACTITUD } \\
\text { INNOVADORA }\end{array}$} & Adoptar innovaciones es una decisión útil & 241 & 5,16 & 1,84 \\
\hline & Valoro a las personas que innovan & 248 & 5,97 & 1,30 \\
\hline & Las personas que son importantes para mí piensan que yo debo innovar & 226 & 4,90 & 1,78 \\
\hline & Estoy motivado para innovar & 247 & 4,57 & 2,15 \\
\hline & Las innovaciones mejoran los resultados de mi explotación & 242 & 5,12 & 1,86 \\
\hline & Innovar merece la pena & 241 & 5,16 & 1,84 \\
\hline
\end{tabular}

Fuente: Elaboración propia. 
Como se puede ver en las puntuaciones, la media más alta es un 6'05 que corresponde a la variable "Me siento corresponsable de los fallos en mi explotación" lo que indica una clara orientación hacia el aprendizaje que permita al productor una mejora continua. Por otra parte, el productor muestra un alto grado de implicación en la explotación ("cuando una nueva técnica/producto no da resultados analizo las causas del fallo", 5'67). Otro aspecto positivo es también la predisposición al aprendizaje mediante la cooperación, aunque a un nivel superficial, con otros productores ("Intercambio experiencias con otros agricultores", 5'95). Resulta ilustrativo que el tándem cooperación/competitividad se incline hacia el lado de la cooperación, estando en línea también con la Estrategia Europea 2020. Esto indica que se percibe como beneficiosa la cooperación. Lo que quiere decir que pesan más las ventajas obtenidas, dejando en un segundo lugar al recelo de la competitividad empresarial. Por otra parte, también es un indicador de la voluntad de aprender. Y aprender se lleva a cabo de la manera más accesible, con productores que trabajan con el mismo producto ya que es habitual a lo largo de la geografía valenciana que existan zonas dedicadas prácticamente al monocultivo de una especie. Aunque bien puede significar que el agricultor echa mano de la información más accesible ante la carencia de otras vías para la transferencia de conocimiento, como veremos a continuación.

Por lo que se refiere a la orientación al cliente, el mercado ocupa un lugar destacado en los planteamientos empresariales ("sigo las orientaciones de calidad que transmite el cliente", 5'77, y "la satisfacción del cliente es el principal objetivo de mi empresa", 5'72). Así se confirma la orientación al mercado que lleva caracterizando a la agricultura valenciana durante décadas y cómo este mercado es el principal demandante de innovaciones, aspecto que es claramente percibido por el agricultor.

Asimismo, las puntuaciones medias más altas reflejan una actitud innovadora que se muestra como aquella que sabe apreciar el esfuerzo que conlleva innovar ("valoro a las personas que innovan", 5'97),
En lo que respecta a los aspectos con puntuaciones más bajas, el productor percibe algunos factores externos como factores que dificultan un entorno propicio para la innovación. El precio de mercado es una variable que el productor no puede controlar (" $m i$ preocupación por ofrecer productos más baratos me da una ventaja sobre otras explotaciones", 3'03) y la respuesta refleja una preocupación pesimista acerca de la competencia vía precios.

Como conclusión se puede destacar la implicación del productor con su actividad y cómo es consciente de la importancia de sus clientes. Cabe añadir que existe una predisposición por parte del productor a implicarse en el proceso innovador, pese a que el entorno socioeconómico del agricultor no es percibido como favorable para tales actuaciones. Bien sea por desconocimiento o por carencia lo cierto es que el productor no percibe que tenga mecanismos públicos o privados en los que pueda apoyarse fácilmente y ve más accesible la información que le proporcionan otros productores.

\subsection{Perfiles innovadores de los agricultores valencianos}

Una vez analizados los estadísticos descriptivos sobre las opiniones de los agricultores, se expone a continuación, un modelo que explica el comportamiento innovador, teniendo en cuenta las características individuales y organizativas que figuran en la Tabla 1. A partir de estos resultados se puede catalogar al productor agrario innovador según las características socioeconómicas que favorecen su inclinación hacia cada uno de los tres factores innovadores estudiados. Se han agrupado (ver Tabla 4) los resultados según el nivel de estudios del encuestado y su localización en medio rural, urbano o intermedio siguiendo los criterios de ruralidad de la OCDE y la clasificación de sistemas territoriales de Boix y Galetto (2008) modificado por López Estornell (2010). 
Tabla 4. Perfiles innovadores según nivel de formación y localización de las explotaciones.

\begin{tabular}{l|l|c|c|c|c} 
& & $\begin{array}{c}\text { Débilmente } \\
\text { innovadores }\end{array}$ & $\begin{array}{c}\text { Innovador } \\
\text { Innovadores }\end{array}$ & $\begin{array}{c}\text { Innor } \\
\text { intermedio (a) }\end{array}$ \\
& $\%$ & $11,7 \%$ & $28,3 \%$ & $27,6 \%$ \\
intermedio (b)
\end{tabular}

Innovador intermedio (a) Actitud innovadora (Baja) y alguna orientación (Alta)

Innovador intermedio (b) Actitud innovadora (Alta) y alguna orientación (Baja)

Fuente: Elaboración propia.

Teniendo en cuenta estos resultados se pueden establecer tres niveles de innovación.

\section{a) Productores agrarios débilmente innovadores}

En este segmento se encuadran aquellos casos que muestran una orientación desfavorable hacia los tres componentes del comportamiento innovador. En nuestra muestra constituyen el $11,7 \%$ de los entrevistados (Tabla 4). Hay que tener en cuenta que los agricultores encuestados son personas que colaboran con organizaciones profesionales por lo que es lógico que el colectivo débilmente innovador no tenga una presencia mayoritaria en la muestra. En la tabla sí se comprueba que en este grupo son mayoritarias las explotaciones donde el entrevistado no dispone de estudios o sólo de estudios básicos. Además, son explotaciones que se localizan en un medio predominantemente rural, lo que pone en evidencia los handicaps existentes en una parte del territorio y la pertinencia de las políticas de desarrollo rural. En este grupo se encuentran muchas explotaciones cuya producción es predominantemente ganadera. Los resultados empresariales también influyen en el comportamiento innovador, de manera que aquellas que no superan los $5.000 €$ anuales de margen bruto aproximado tienen una probabilidad mayor de estar presentes en esta categoría. También el modelo de agricultor a tiempo parcial está presente en este perfil.

\section{b) Productores agrarios Innovadores}

En este perfil se incluyen aquellos agricultores que muestran una orientación favorable hacia los tres componentes del comportamiento innovador (Orientación al Mercado, Orientación al Aprendizaje, y Actitud Innovadora). Se observa que, en este grupo, cuya participación en el total de encuestados es del 28,3\%, existen características opuestas al grupo anterior, con presencia mayoritaria (aunque no exclusiva) de agricultores con estudios más allá de los básicos y localización en zonas urbanas. Este grupo cuenta con una mayor presencia relativa de explotaciones cuya producción se dedica a cultivos hortícolas o cítricos. Contar con infraestructura para riego por goteo también posiciona a las explotaciones en el grupo de innovadores. En cuanto a los resultados empresariales, en contraposición a los pertenecientes al grupo de débilmente innovadores, presentar un margen bruto anual superior a 50.000€ es una característica que influye positivamente para posicionarse en el grupo de explotaciones innovadoras.

En cuanto a las características sociodemográficas del productor, el productor "tipo" de esta categoría dedica más de la mitad de su tiempo a la explotación y ha recibido formación. Las explotaciones que tienen toda o parte de su suelo en régimen de arrendamiento muestran una orientación positiva hacia esta categoría. Es destacable que pertenecer a cooperativas también influye positivamente en el comportamiento innovador, de manera que los productores que pertenecen a alguna asociación en la muestra tienden a mostrar no sólo una mejor actitud innovadora, sino mejores orientaciones al aprendizaje y al mercado. 


\section{c) Productores agrarios Innovadores intermedios}

En esta categoría se encuadran dos posiciones respecto a su actitud innovadora y orientación que aparecen encontradas. Por tanto, son explotaciones que presentan un posicionamiento favorable en algunos de los constructos sin llegar a ser favorable para todos ellos. Es llamativo que una de estas categorías "Innovador intermedio (b)" es la mayoritaria (un $32,4 \%$ de los encuestados), con agricultores con una actitud innovadora apreciable pero relativamente baja orientación al mercado o al aprendizaje. En este caso, el problema no es la propensión para asumir cambios, sino a percibir las señales del mercado, incluso cuando el nivel de formación mayoritario (un 63\%) es superior al básico y su localización es mayoritariamente urbana. Aquí se detecta la carencia de unos prerrequisitos que la política agraria y los sistemas de formación deberían de contrarrestar. En el "Innovador intermedio (a)" se confirma que un nivel menor de formación y la lejanía a zonas urbanas no están asociadas a una mayor actitud innovadora. En este grupo tienden a tener una mayor presencia relativa explotaciones orientadas a cultivos leñosos, cuya fijeza puede explicar en parte la baja propensión a innovar. Se observa que en este grupo la edad del productor tiende a ser mayor, lo que influye negativamente sobre todo en la orientación al aprendizaje y en la actitud innovadora.

A modo de resumen, a continuación se presenta una figura que ilustra los perfiles que se pueden establecer.

Figura 3. Perfiles de agricultores según su posición en los tres factores de innovación identificador: Orientación al Mercado, Orientación al Aprendizaje y Actitud Innovadora.

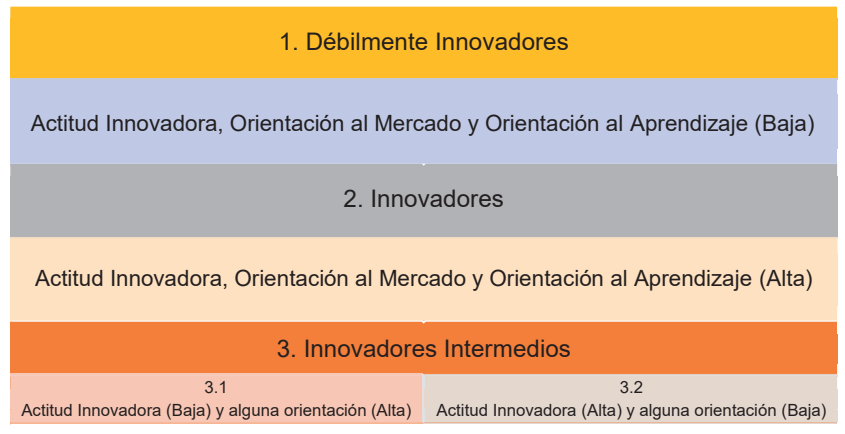

Fuente: Elaboración propia.

\section{CONCLUSIONES}

En términos generales la presente investigación ha dotado al conocimiento de un nuevo punto de referencia para avanzar en el estudio de la innovación en el sector agrario valenciano.

Los resultados de la investigación muestran las características socioeconómicas del productor y su explotación que más favorecen el comportamiento innovador, entendido éste como la combinación de tres componentes: la orientación al mercado, la orientación al aprendizaje y actitud innovadora. En nuestra encuesta existe una alta presencia de agricultores con actitud innovadora, que no consideramos representa necesariamente al agricultor valenciano, pero si denota qué características tienen los agricultores más innovadores.

Es interesante constatar que la actitud innovadora se ve muy asociada al nivel de formación: a mayor educación mejor predisposición muestra el productor a adoptar una actitud innovadora. Por tanto, los resultados, hacen hincapié en la necesidad de contar con responsables empresariales profesionales lo que, en zonas rurales, hoy por hoy, supone un handicap. Son aspectos que las políticas educativas y de desarrollo rural deberían tener en cuenta.

El estudio demuestra que los productores agrarios más implicados en la actividad son los que realmente adoptan una actitud fructífera para conseguir que el sector se integre plenamente en la dinámica económica actual. Así pues, el productor innovador es aquel que muestra implicación en su actividad y lo demuestra dedicándole más de la mitad de su tiempo a la explotación. También favorece el comportamiento innovador el que toda o parte de la explotación se encuentre en régimen de arrendamiento. De igual manera favorece la innovación el contar con mano de obra asalariada.

Todos estos factores indican el intento por parte del sector de paliar uno de los problemas estructurales del sector como es la agricultura a tiempo parcial. A lo largo de las décadas la deriva de la agricultura hacia este modelo productivo permitió mantener la estructura 
minifundista y que el productor adoptase una postura diferente respecto a la explotación, asumiendo otras ocupaciones como su actividad principal. El minifundismo también es un aspecto que va asociado a la agricultura parcial, y con ellos la desprofesionalización del sector. Asumir el régimen de arrendamiento para la explotación puede ser un reflejo de cómo se intenta alcanzar un tamaño necesario para aprovechar las economías de escala, lo que evidentemente precisa de un productor profesional enteramente dedicado a la actividad. La agricultura a tiempo parcial también tuvo como consecuencia el auge de organizaciones cuyo objetivo era proveer los servicios como consecuencia de la desprofesionalización del sector, ya que el productor veía posible llevar a cabo la actividad agraria compaginándola con la actividad en otros sectores. Sobre este aspecto, el pertenecer a una organización es un aspecto que, en nuestra investigación, favorece la innovación, sobre todo en el caso de las cooperativas o SATs. De esta forma, se alinean con la Estrategia Europea 2020 con el establecimiento de vínculos interorganizacionales. Así, podemos constatar que la estructura organizacional valenciana nació y floreció con un objetivo, y hoy en día es un factor sumamente útil para mantener a la agricultura valenciana en la economía moderna. No obstante, no hay que olvidar que la agricultura parcial funcionó en su momento dando salida a problemas como este, que son consecuencia de las carencias estructurales del sector, pero en la actualidad dejamos constancia de la necesidad de profesionalizar la agricultura valenciana.

Por otra parte, según la orientación productiva vemos que las explotaciones que se dedican al cítrico o al cultivo de hortícolas tienden a situarse en el grupo de innovadoras. Puede reforzar esta impresión el hecho de que sean sectores muy orientados al mercado, con una vertiente exportadora notable. Pero, por otra parte, las explotaciones que cuentan con una infraestructura de riego también son explotaciones con predisposición innovadora. Este hecho puede poner de relieve que la agricultura litoral se diferencia de la agricultura de interior, apareciendo la litoral más integrada en la dinámica económica actual. Ello sugiere la necesidad de adoptar políticas de desarrollo rural que revitalicen la diversificación económica en zonas de interior, teniendo en cuenta también el reto de la sostenibilidad.
Por último, en el grupo innovador están muy presentes explotaciones que pertenecen que obtienen un margen bruto anual superior a $50.000 €$ anuales. Esta tendencia muestra la inseguridad financiera que padece el sector e ilustra el hecho de que se asocie el adoptar innovaciones con la inversión de dinero. No queremos decir con esto que ser pequeña condene la explotación a ser débilmente innovadora, pues es un conjunto de características las que acaban influyendo en el comportamiento innovador.

En resumen, se ha podido observar que las carencias estructurales que fueron objeto de estudio a finales del siglo XX continúan presentes en la agricultura valenciana, si bien es cierto que se puede observar una tímida evolución con el propósito de paliarlas. Con todo ello, el contenido del presente trabajo evidencia el esfuerzo por parte del productor valenciano innovador de hacer frente a los problemas tradicionales que aquejan al sector. La Educación aparece como uno de los factores clave sobre el cual hay que seguir incidiendo para hacer viable el sector. Este trabajo puede arrojar luz a la hora de realizar políticas activas de promoción de la innovación agraria ya que señala con claridad las variables sobre las que cabe incidir para mejorar la eficacia de los esfuerzos públicos sobre el sector.

\section{REFERENCIAS BIBLIOGRÁFICAS}

Agriresearch factsheet on enhancing human capital and boosting innovation systems. Accesible en: https:// ec.europa.eu/info/sites/info/files/food-farming-fisheries/ farming/documents/factsheet-agri-human-capital_ en.pdf. (27-diciembre 2020)

Aguilar, E., Amaya-Corchuelo, S., y López-Moreno, I. (2016). "Alimentos con calidad. Nuevas estrategias rurales para nuevos consumidores". Arxiu d'Etnografia de Catalunya 16: 137-152. https://doi.org/10.17345/aec16.137-152

Alba, M. F., López-García Usach, T. García Álvarez-Coque, J.M. y Mas Verdú, F. (2010). Conocimiento incorporado y vínculos sectoriales. El caso de una región con baja capacidad de absorción. International meeting on regional science. The future of cohesion policy. XVI reunión de estudios regionales. AECR. Badajoz, del 17 al 19 de noviembre de 2010. Recuperado, 1 de diciembre de 2018: https://old.aecr.org/web/congresos/2010/ htdocs/pdf/p172.pdf

Albisu Aguado, L. M. (2019). Las interacciones institucionales como condicionante del desarrollo de la innovación en el sector agroalimentario.

Amaya-Corchuelo, S., Fernández Zarza, M., \& Aguilar Criado, E. (2018). Placer, salud y sociabilidad. El hecho 
alimentario a través del jamón ibérico. Disparidades. Revista De Antropología, 73(2), 425-452. https://doi. org/10.3989/rdtp.2018.02.008

Arnalte Alegre, E. (1989). Estructura de las explotaciones agrarias y externalización del proceso productivo: implicaciones para el debate sobre el proteccionismo. Información Comercial Española, 666, 101-117

Boix, R. y Galleto, V. (2008). Marshallian Industrial districts in Spain. Scienze Regionali, 7 (3), 29-52.

Connor, J.M. y Schiek, W.A. (1997). Food processing: an industrial owerhouse in transition. New York: John Wiley \& Sons ( $2^{\mathrm{a}}$ ed.).

Corbetta, P. (2003). Metodología y técnicas de investigación social. Madrid: McGraw Hill.

Cordovés Torres Gómez de Cádiz, D., Franch León, K., Zenea Montejo, M., Díaz Fernández, I., \& Toscano Ruiz, D. F. (2017). La gestión de la innovación agraria: una mirada teórico-práctica. Cofin Habana, 11(1), 1-15.

de Cádiz, D. C. T. G., Montejo, M. Z., \& Ramiro, C. J. M. (2020). Perspectiva teórica de la gestión de la innovación agraria/Theoretical Perspective on Managing Innovation in Agriculture. Revista Economía y Desarrollo (Impresa), 163(1).

de la Fuente Cabrero, C., Segovia-Pérez, M., \& FigueroaDomecq, C. (2014). Implications of Financial Institution Support for Women's Business Projects. ESIC Market, 149. https://doi.org/10.7200/esicm.149.0453.3

Instituto Nacional de Estadística. Censo Agrario (19992009). Recuperado: http://www.ine.es

López-Estornell, M. (2010). Empresa Innovadora, Conocimiento y Distrito Industrial. Tesis Doctoral. Valencia: Universitat Politècnica de València.

López-García Usach, T. (2013). Comportamiento innovador del sistema agroalimentario en la comunidad valenciana: cadena de valor, vínculos intersectoriales y territorio. Tesis doctoral. Valencia: Universitat Politècnica de València.

Peraza Castaneda, E. H., Gómez García, J. M., \& Aleixandre Mendizábal, G. (2016). Los factores determinantes del comportamiento innovador de las cooperativas: un análisis para el caso de Castilla y León. REVESCO. Revista de Estudios Cooperativos, (122), 252-284. https://doi.org/10.5209/rev_REVE.2016.v122.52025

Pérez Ledo, P. (2018). Educación para la innovación agraria. Un modelo para la acción educativa. CEIR. Creativity and Educational Innovation Review, 2, 128-143. https:// doi.org/10.7203/CREATIVITY.2.13756

Pérez-Gil, J. A., Chacón, S. y Moreno, R., (2000). Validez de constructo: el uso de análisis factorial exploratorioconfirmatorio para obtener evidencias de validez. Psicothema, 12, 2, 422-446.
Pomering, A. (2017). Marketing for sustainability: Extending the conceptualisation of the marketing mix to drive value for individuals and society at large. Australasian Marketing Journal (AMJ), 25(2),157-165.https://doi. org/10.1016/j.ausmj.2017.04.011

Salas Arbeláez, L., García Solarte, M., \& Azuero Rodríguez, A. R. (2018). Género del gerente e innovación: Caso empírico en Pymes de Cali. Revista investigación desarrallo innovación, 8 (2), 223-235. doi: 10.19053/20278306. v8.n2.2018.7962

Savall, N. V., Pizarro, J. E., \& López, D. E. V. (2020). Redes de apoyo al emprendimiento femenino en territorios rurales. OBETS. Revista de Ciencias Sociales, 15(1), 317 356 https://doi.org/10.14198/OBETS2020.15.1.11

Segovia-Pérez, M., Santero Sánchez, R., Domecq, C. F., \& Núñez, R. B. C. (2019). Caracterización de la brecha salarial en el turismo rural español: una comparativa con el entorno laboral turístico urbano. Ager: Revista de estudios sobre despoblación y desarrollo rural= Journal of depopulation and rural development studies, (26), 7-34.

Yagüe-Perales, R. M., Perez-Ledo, P., \& March-Chorda, I. (2020). The unexpected profile of agricultural innovators: evidence from an empirical study. New Medit: Mediterranean Journal of Economics, Agriculture and Environment= Revue Méditerranéenne d'Economie Agriculture et Environment, 19(2). https://doi. org/10.30682/nm2002f.

\section{NOTAS BIOGRÁFICAS}

\section{PAU PÉREZ-LEDO}

Sociólogo por la Universitat d'Alacant y en la actualidad es profesor asociado en el Departamento Sociología i Antropología Social de la Universitat de València y en el Departamento economía y Ciencias Sociales de la Universitat Politècnica de València. Sus áreas de interés son la educación, la agricultura, la innovación y el turismo.

\section{MÓNICA SEGOVIA-PÉREZ}

Socióloga, profesora Titular en la Universidad Rey Juan Carlos de Madrid y coordinadora del Grado de Sociología en la misma Universidad. Sus áreas de interés son la innovación-tecnología, el género y el turismo donde cuenta con diferentes publicaciones con índices de calidad relativos. 